\title{
Probing Newton's constant on vast scales: Dvali-Gabadadze-Porrati gravity, cosmic acceleration, and large scale structure
}

\author{
Arthur Lue* \\ Center for Education and Research in Cosmology and Astrophysics, Department of Physics, Case Western Reserve University, \\ Cleveland, Ohio 44106-7079, USA \\ and CERN Theory Division, CH-1211 Geneva 23, Switzerland \\ Román Scoccimarro ${ }^{\dagger}$ \\ Center for Cosmology and Particle Physics, Department of Physics, New York University, New York, New York 10003, USA \\ Glenn D. Starkman \\ Center for Education and Research in Cosmology and Astrophysics, Department of Physics, Case Western Reserve University, \\ Cleveland, Ohio 44106-7079, USA \\ and CERN Theory Division, CH-1211 Geneva 23, Switzerland
}

(Received 9 February 2004; published 18 June 2004)

\begin{abstract}
The nature of the fuel that drives today's cosmic acceleration is an open and tantalizing mystery. The brane-world theory of Dvali, Gabadadze, and Porrati (DGP) provides a context where late-time acceleration is driven not by some energy-momentum component (dark energy), but rather is the manifestation of the excruciatingly slow leakage of gravity off our four-dimensional world into an extra dimension. At the same time, DGP gravity alters the gravitational force law in a specific and dramatic way at cosmologically accessible scales. We derive the DGP gravitational force law in a cosmological setting for spherical perturbations at subhorizon scales and compute the growth of large-scale structures. We find that a residual repulsive force at large distances gives rise to a suppression of the growth of density and velocity perturbations. Explaining the cosmic acceleration in this framework leads to a present day fluctuation power spectrum normalization $\sigma_{8}$ $\leqslant 0.8$ at about the two-sigma level, in contrast with observations. We discuss further theoretical work necessary to go beyond our approximations to confirm these results.
\end{abstract}

DOI: 10.1103/PhysRevD.69.124015

PACS number(s): 04.50.+h

\section{INTRODUCTION}

The discovery of a contemporary cosmic acceleration $[1,2]$ is one of the most profound scientific observations of the 20th century. We are now challenged to answer the open and tantalizing question of what drives that acceleration. While a conventional explanation exists (i.e. dark energysome new negative-pressure energy-momentum component), an intriguing line of thought is gaining attention: the accelerated expansion is not a result of yet another ingredient in our already gunky cosmic gas tank, but rather is a signal of our lack of understanding of gravitational physics on large scales [3-14].

Being able to observationally differentiate the two possibilities, dark energy versus modified gravity, is an essential component in developing the modified-gravity paradigm. One can easily envision some modified-gravity model leading to an expansion history that can be identically reproduced by some dark-energy model. Thus, observations that depend only on anomalous expansion histories are insufficient to tease out the acceleration's root cause. However, as we argue in Ref. [15], if one attempts to modify cosmology at today's Hubble scale, $H_{0}$, through altering the equations

\footnotetext{
*Email address: lue@cern.ch

†Email address: rs123@nyu.edu

‡Email address: glenn.starkman@cern.ch
}

governing gravitational dynamics, then generically one expects that the gravitational force law of an isolated mass source is altered even at distance scales much smaller than $H_{0}^{-1}$. This effect can then be exploited to differentiate between a modified-gravity explanation of today's cosmic acceleration and dark energy (where the gravitational force law remains unaltered) $[15,16]$.

A leading contender in modified-gravity explanations of acceleration is the brane-world theory of Dvali, Gabadadze, and Porrati (DGP). In this theory, gravity appears four dimensional at short distances but is altered at distances large compared to some freely adjustable crossover scale $r_{0}$ through the slow evaporation of the graviton off our fourdimensional brane-world universe into an unseen, yet large, fifth dimension [17-19]. DGP gravity provides an alternative explanation for today's cosmic acceleration [3,4]: just as gravity is conventional four-dimensional gravity at short scales $\left(r \ll r_{0}\right)$ and appears five dimensional at large distance scales $\left(r \gg r_{0}\right)$, so too the Hubble scale, $H(t)$, evolves by the conventional Friedmann equation at high Hubble scales but saturates at a fixed value as $H(t)$ approaches $r_{0}^{-1}$. Thus, if one were to set $r_{0} \simeq H_{0}^{-1}$, where $H_{0}$ is today's Hubble scale, then DGP gravity could account for today's cosmic acceleration in terms of the existence of extra dimensions and a modification of the laws of gravity. The resulting cosmic expansion history is specific and may be tested using a variety of cosmological observations [4,20-22]. However, can 
we distinguish between DGP gravity and a dark energy model that mimics the same cosmic expansion history?

We would naively expect not to be able to probe the extra dimension at distances much smaller than the crossover scale $r_{0}=H_{0}^{-1}$. However, in DGP, although gravity is four dimensional at distances shorter than $r_{0}$, it is not four-dimensional Einstein gravity-it is augmented by the presence of an ultralight gravitational scalar. One only recovers Einstein gravity in a subtle fashion [23-26], and a marked departure from Einstein gravity persists down to distances much shorter than $r_{0}$. For example, for $r_{0} \approx H_{0}^{-1}$ and a central mass source of Schwarzschild radius $r_{g}$, significant and cosmologically sensitive deviations from Einstein gravity occur at distances greater than $[25-28]$

$$
r_{*}=\left(r_{g} r_{0}^{2}\right)^{1 / 3} \approx\left(\frac{r_{g}}{H_{0}^{2}}\right)^{1 / 3} .
$$

Thus a marked departure from conventional physics persists down to scales much smaller than the distance at which the extra dimension is naively hidden, or for our discussion here, the distance at which the Friedmann equation was modified to account for accelerated cosmic expansion. This alteration of gravitational interactions provides a way of differentiating between DGP gravity and dark energy models, and is consistent with the argument we put forth in Ref. [15].

Imminent solar system tests have been shown to be capable of probing the residual deviation from fourdimensional Einstein gravity at distances well below $r_{*}$ $[27,28]$. Nevertheless, it would be ideal to test gravitational physics where dramatic differences from Einstein gravity are anticipated. A detailed study of large scale structure in the Universe can provide such tests of gravitational physics at large distance scales. Unfortunately, prior analyses related to modified-gravity explanations of cosmic acceleration $[15,16]$ are not applicable here. The modified force law is, in effect, sensitive to the background cosmological expansion, since this expansion is intimately tied to the extrinsic curvature of the brane [3,29], and this curvature controls the effective Newtonian potential. A more careful analysis must be performed. In the next section we briefly review DGP gravity and identify the force law necessary to calculate how large scale structure evolves in this cosmological model. We then proceed and compare those results to the standard cosmology, as well as to a cosmology that exactly mimics the DGP expansion history using dark energy. Finally, we discuss the observational implications of our results on the growth of structure in DGP gravity and conclude with some remarks and a discussion of future work needed to improve upon our treatment.

\section{DGP GRAVITY}

We review the important points of the DGP brane-world model, including cosmology. We then modify the calculation performed in Ref. [27] to determine the gravitational force law in an evolving cosmological background, rather than in a static background de Sitter space. This calculation is the re- sult in this paper that allows one to answer questions of cosmological interest developed in the next sections.

\section{A. DGP model}

Consider a brane-world theory of gravity (one in which ordinary particles and fields, other than the graviton, are confined to a three-dimensional hypersurface-the braneembedded in a higher dimensional space - the bulk) with an infinite-volume bulk and a metastable brane graviton [17]. We take a four-dimensional brane world embedded in a fivedimensional Minkowski spacetime. The bulk is empty; all energy momentum is isolated on the brane. The action is

$$
S_{(5)}=-\frac{1}{16 \pi} M^{3} \int d^{5} x \sqrt{-g} R+\int d^{4} x \sqrt{-g^{(4)}} \mathcal{L}_{m}+S_{G H} .
$$

The quantity $M$ is the fundamental five-dimensional Planck scale. The first term in Eq. (2.1) corresponds to the EinsteinHilbert action in five dimensions for a five-dimensional metric $g_{A B}$ (bulk metric) with Ricci scalar $R$. The term $S_{G H}$ is the Gibbons-Hawking action. In addition, we consider an intrinsic curvature term which is generally induced by radiative corrections by the matter density on the brane [17]:

$$
-\frac{1}{16 \pi} M_{P}^{2} \int d^{4} x \sqrt{-g^{(4)}} R^{(4)} .
$$

Here, $M_{P}$ is the observed four-dimensional Planck scale (see Refs. [17-19] for details). Similarly, Eq. (2.2) is the Einstein-Hilbert action for the induced metric $g_{\mu \nu}^{(4)}$ on the brane, $R^{(4)}$ being its scalar curvature. The induced metric is ${ }^{1}$

$$
g_{\mu \nu}^{(4)}=\partial_{\mu} X^{A} \partial_{\nu} X^{B} g_{A B},
$$

where $X^{A}\left(x^{\mu}\right)$ represents the coordinates of an event on the brane labeled by $x^{\mu}$. The action given by Eqs. (2.1) and (2.2) leads to the following equations of motion:

$$
\frac{1}{2 r_{0}} G_{A B}+\delta(\text { brane }) G_{A B}^{(4)}=\left.\frac{8 \pi}{M_{\mathrm{P}}^{2}} T_{A B}\right|_{\text {brane }},
$$

where $G_{A B}$ is the bulk Einstein tensor, $G_{A B}^{(4)}$ is the Einstein tensor of the induced brane metric, and $\left.T_{A B}\right|_{\text {brane }}$ is the matter energy-momentum tensor on the brane. We have defined a crossover scale

$$
r_{0}=\frac{M_{P}^{2}}{2 M^{3}}
$$

This scale characterizes that distance over which metric fluctuations propagating on the brane dissipate into the bulk [17].

\footnotetext{
${ }^{1}$ Throughout this paper, we use $A, B, \ldots=\{0,1,2,3,5\}$ as bulk indices, $\mu, \nu, \ldots=\{0,1,2,3\}$ as brane spacetime indices, and $i, j, \ldots=\{1,2,3\}$ as brane spatial indices.
} 


\section{B. Cosmological background}

Let us review some important details of the cosmological background, for a general expansion of a spatially flat brane into a Minkowski-flat bulk. We are primarily interested in the late evolution of the Universe, in particular the matterdominated era where the energy-momentum content of the Universe is well-represented by a pressureless distribution of galaxies, spatially homogeneous on the largest scales. The spatially homogeneous cosmological background of such a Universe is driven by energy-momentum given by

$$
\left.T_{B}^{A}\right|_{\text {brane }}=\delta(z) \operatorname{diag}(\rho(\tau), 0,0,0,0),
$$

with spacetime geometry dictated by the line element [3]

$$
\begin{aligned}
d s^{2}= & \left(1 \mp \frac{\ddot{\bar{a}}}{\dot{\bar{a}}}|z|\right)^{2} d \tau^{2}-\bar{a}^{2}(\tau)\left(1 \mp \frac{\dot{\bar{a}}}{\bar{a}}|z|\right)^{2}\left[\delta_{i j} d \lambda^{i} d \lambda^{j}\right] \\
& -d z^{2}
\end{aligned}
$$

Here, dot refers to differentiation with respect to the cosmological time $\tau$, the coordinate $z$ is the extra dimension, and the brane scale factor, $\bar{a}(\tau)$ satisfies a modified Friedmann equation

$$
H^{2} \pm \frac{H}{r_{0}}=\frac{8 \pi}{3 M_{P}^{2}} \rho(\tau)
$$

where $H(\tau)=\dot{\bar{a}} / \bar{a}$. The two choices of sign represent two distinct cosmological phases. The phase of interest (the selfaccelerating phase) corresponds to the lower sign, but we keep both for the sake of completeness.

This Friedmann equation (2.8) already makes the theory distinct from standard $\Lambda \mathrm{CDM}$ (cold dark matter with cosmological constant) cosmology, and observational signatures constraining DGP cosmology have been considered in e.g. Refs. [4,21,22]. Using constraints from type 1A supernovae [21], the best fit $r_{0}$ is $r_{0}=1.21_{-0.09}^{+0.09} H_{0}^{-1}$, where $H_{0}$ is today's Hubble scale. Taking $H_{0} \approx 70 \mathrm{~km} \mathrm{~s}^{-1} \mathrm{Mpc}^{-1}$, it implies $r_{0}$ $\approx 5 \mathrm{Gpc}$.

However, we wish to focus on those properties of DGP gravity that are affected by the modification of the force law, and cannot be mimicked by some dark energy component that produced the same expansion history. Following this program, we focus particularly at distance scales much smaller than the Hubble radius, $H^{-1}$. As described in the Introduction, the gravitational force law is significantly different from four-dimensional Einstein, even at these short distance scales. We wish to determine the form of the corrections in the background of the expected matter-dominated cosmology, Eq. (2.8).

We are concerned with processes at distances, $r$, such that $r H \ll 1$. Under that circumstance it is useful to change coordinates to a frame that surrenders explicit brane spatial homogeneity but preserves isotropy

$$
\begin{aligned}
& r\left(\tau, \lambda^{i}\right)=\bar{a}(\tau) \lambda \\
& t\left(\tau, \lambda^{i}\right)=\tau+\frac{\lambda^{2}}{2} H(\tau) \bar{a}^{2}(\tau),
\end{aligned}
$$

for all $z$ and where $\lambda^{2}=\delta_{i j} \lambda^{i} \lambda^{j}$. The line element becomes

$$
\begin{aligned}
d s^{2}= & {\left[1 \mp 2(H+\dot{H} / H)|z|-\left(H^{2}+\dot{H}\right) r^{2}\right] d t^{2} } \\
& -[1 \mp 2 H|z|]\left[\left(1+H^{2} r^{2}\right) d r^{2}+r^{2} d \Omega\right]-d z^{2},
\end{aligned}
$$

where here dot represents differentiation with respect to the time coordinate, $t$. Moreover, $H=H(t)$ in this coordinate system. All terms of $\mathcal{O}\left(r^{3} H^{3}\right)$ or $\mathcal{O}\left(z^{2} H^{2}, z H r H\right)$ and higher have been neglected.

The coordinate system in Eq. (2.11) will be the most useful when considering the cosmological scenarios we are interested in. One can see that the bulk is a Rindler space. This has a fairly natural interpretation if one imagines the bulk picture [3,29]. One imagines riding a local patch of the brane, which appears as hyperspherical surface expanding into (or away from) a five-dimensional Minkowski bulk. This surface either accelerates or decelerates in its motion with respect to the bulk, creating a Rindler-type potential.

\section{Nonrelativistic matter sources}

We are interested in finding the metric for compact, spherically symmetric overdensities in the background of a matter-dominated cosmology. Because we are only concerned with distance scales such that $r H \ll 1$, then to leading order in $r^{2} H^{2}$ and $z H$, the solutions to the field equations (2.4) are also solutions to the static equations, i.e. the metric is quasistatic, where the only time dependence comes from the slow evolution of the extrinsic curvature of the brane. To be explicit, we are looking at the nonrelativistic limit, where the metric, or the gravitational potentials, of a matter source depends only on the instantaneous location of its elements, and not on the motion of those elements.

Under this circumstance, one can choose a coordinate system in which the cosmological metric respects the spherical symmetry of the matter source. Let the line element be

$$
\begin{aligned}
d s^{2}= & N^{2}(t, r, z) d t^{2}-A^{2}(t, r, z) d r^{2} \\
& -B^{2}(t, r, z)\left[d \theta^{2}+\sin ^{2} \theta d \phi^{2}\right]-d z^{2} .
\end{aligned}
$$

We are interested in small deviations of the metric from flatness so we define functions $\{n(t, r, z), a(t, r, z), b(t, r, z)\}$ such that

$$
\begin{aligned}
& N(t, r, z)=1+n(t, r, z) \\
& A(t, r, z)=1+a(t, r, z) \\
& B(t, r, z)=r[1+b(t, r, z)] .
\end{aligned}
$$

The key is that because we are interested primarily in phenomena whose size is much smaller than the cosmic horizon, the effect of cosmology is almost exclusively to control the 
extrinsic curvature, of the brane. This can be interpreted as a modulation of the brane's stiffness or the strength of the scalar gravitational mode.

We take the energy-momentum tensor to now be

$$
\left.T_{B}^{A}\right|_{\text {brane }}=\delta(z) \operatorname{diag}(\rho(t)+\delta \rho(t, r), 0,0,0,0),
$$

where the source mass is an overdensity of compact support (i.e. its extent is some radius, $R \ll H^{-1}$ ). Given a source mass whose overdensity with respect to the cosmological background goes as $\delta \rho(r, t)$, we may define an effective Schwarzschild radius

$$
R_{g}(r, t)=\frac{8 \pi}{M_{P}^{2}} \int_{0}^{r} r^{2} \delta \rho(r, t) d r .
$$

We solve the perturbed Einstein equations in quasistatic approximation by generalizing the method used in Ref. [27], obtaining the metric of a spherical mass overdensity $\delta \rho(t, r)$ in the background of the cosmology described by Eq. (2.11) (rather than de Sitter space). The metric on the brane, using the residual gauge fixing $\left.b(t, r)\right|_{z=0}=0$, is then given by

$$
\begin{gathered}
\left.r n^{\prime}(t, r)\right|_{z=0}=\frac{R_{g}}{2 r}[1+\Delta(r)]-\left(H^{2}+\dot{H}\right) r^{2} \\
\left.a(t, r)\right|_{z=0}=\frac{R_{g}}{2 r}[1-\Delta(r)]+\frac{1}{2} H^{2} r^{2}
\end{gathered}
$$

where dot now denotes differentiation with respect to $t$ and prime denotes differentiation with respect to $r$. Note that the background contribution is included in these metric components. The quantity $\Delta(r)$ is defined as

$$
\Delta(r)=\frac{3 \beta r^{3}}{4 r_{0}^{2} R_{g}}\left[\sqrt{1+\frac{8 r_{0}^{2} R_{g}}{9 \beta^{2} r^{3}}}-1\right],
$$

and

$$
\beta=\frac{1 \pm 2 r_{0} H+2 r_{0}^{2} H^{2}}{1 \pm 2 r_{0} H}
$$

Though it is not of explicit interest here, the full $z$ dependence of the metric may be deduced from Eqs. (2.16) and (2.17) using equations laid out in the appendix of Ref. [27] with trivial alterations accounting for the differing cosmological background.

The result Eqs. (2.16)-(2.19) is valid for $r \ll H^{-1}$ and $r$ $\ll r_{0}$, but only if the spatial support of $\delta \rho(r, t)$ extends only to radii much less than $H^{-1}$, so that there is a clear distinction between the matter making up the overdensity and the cosmological background. The result is virtually identical to the strictly static de Sitter background case except there $\beta$ $=1 \pm 2 r_{0} H$. One may also confirm that in the absence of perturbations (i.e., $\delta \rho$ or $R_{g}=0$ ), the background metric Eq. (2.11) is a consistent, quasistatic solution. This point is analogous to the well-known idea that one may reproduce the Friedmann equation in matter-dominated cosmologies with just the Newtonian interaction between matter particles.
From inspection of Eq. (2.19), we see that, in addition to $r_{0}$, there exists a new transition scale

$$
r_{*}=\left[\frac{r_{0}^{2} R_{g}}{\beta^{2}}\right]^{1 / 3},
$$

such that when $r \ll r_{*}$, the Einstein phase, the metric functions on the brane reduce to

$$
\begin{aligned}
& n=-\frac{R_{g}}{2 r} \pm \sqrt{\frac{R_{g} r}{2 r_{0}^{2}}} \\
& a=\frac{R_{g}}{2 r} \mp \sqrt{\frac{R_{g} r}{8 r_{0}^{2}}} .
\end{aligned}
$$

When $r \gg r_{*}$, the weak-brane phase, the metric functions on the brane become

$$
\begin{aligned}
& n=-\frac{R_{g}}{2 r}\left[1+\frac{1}{3 \beta}\right]-\frac{1}{2}\left(H^{2}+\dot{H}\right) r^{2} \\
& a=\frac{R_{g}}{2 r}\left[1-\frac{1}{3 \beta}\right]+\frac{1}{2} H^{2} r^{2} .
\end{aligned}
$$

In this phase, the extra scalar mode, the would-be radion, alters the effective Newton's constants for the gravitational potentials represented by $n(t, r)-\left.n(t, r)\right|_{\text {background }}$, the Newtonian potential, and $a(t, r)-\left.a(t, r)\right|_{\text {background }}$, the gravitomagnetic potential.

One may simply check that the full $(t, r, z)$-dependent metric satisfies the complete modified Einstein equations (2.4) to the desired order. Note that to this order of precision, the velocity of the matter distribution $\delta \rho(t, r)$ does not affect spacetime geometry (until order $v^{2}$ or $v r H$ ), thus corroborating the quasistatic approximation.

\section{Caveats}

The approximations $v \ll 1$ ( $v$ represents peculiar matter velocities) and $r H \ll 1$ play a role in several places and allow a series of crucial simplifications that need to be spelled out. These two approximations are lumped together because the Hubble-flow velocity and peculiar velocities play almost identical roles in the relevant field equations. The following are the operational simplifications:

(i) Nonrelativistic, quasistatic sources. Source-velocity dependent contributions to the gravitational field are subleading. One may use the static Einstein equations and still be assured that the metric on the brane is accurate to $\mathcal{O}\left(v^{2}, r^{2} H^{2}\right)$.

(ii) Near-field regime. Related to the above simplification, the source evolves slowly enough that radiative effects are negligible at these radii. We may safely avoid scalar radiation on the brane and gravity-wave evaporation into the bulk and other radiative bulk effects $[29,30]$. These radiative scalar modes may also have classical instabilities that might become relevant outside this regime [31]. 
(iii) Resolving background matter from perturbation. This simplification is specific to DGP gravity. The metric components Eqs. (2.16) and (2.17) depends on the background Hubble expansion, and the calculation crucially depends on the assumption that the overdensity does not alter the background cosmology. This can been seen to be self-consistent in the DGP field equations only when the radius of support for the overdensity is much smaller than the Hubble radius, $H^{-1}$.

(iv) Geodesic motion is Newtonian, i.e. the geodesic equation reduces to Newton's second law where the potential is $g_{00}$ or $n(t, r)$.

If we stray too far from the assumptions $v \ll 1$ and $r H \ll 1$, then effects safely disregarded may start intruding into and complicating the analysis, particularly when $\mathrm{H} \sim \mathrm{H}_{0}$, introducing additional effects of equal significance to the ones included here.

\section{GROWTH OF DENSITY PERTURBATIONS}

\section{A. Spherical perturbations}

Let us consider the evolution of a spherical top-hat perturbation $\delta(t, r)$ of top-hat radius $R_{t}$, where $\rho(t, r)=\bar{\rho}(t)(1$ $+\delta)$ is the full density distribution and $\bar{\rho}(t)$ is the background density. At subhorizon scales $(H r \ll 1)$, the contribution from the Newtonian potential, $n(t, r)$, dominates the geodesic evolution of the overdensity. From Eq. (2.23) it follows that the equation of motion for the perturbation $\delta$ is

$$
\begin{aligned}
& \ddot{\delta}-\frac{4}{3} \frac{\dot{\delta}^{2}}{1+\delta}+2 H \dot{\delta} \\
& \quad=4 \pi G \bar{\rho} \delta(1+\delta)\left[1+\frac{2}{3 \beta} \frac{1}{\epsilon}(\sqrt{1+\epsilon}-1)\right],
\end{aligned}
$$

where the definition of $\epsilon \equiv 8 r_{0}^{2} R_{g} / 9 \beta^{2} R_{t}^{3}$ follows from the identification of the expression in square brackets with 1 $+\Delta(r)$ [see Eq. (2.18)], and we have restricted ourselves to the self-accelerating branch (i.e., the lower sign choice in all equations in the previous section).

For clarity, we may recast the time evolution of $\beta$ and $\epsilon$ in terms of $\delta$ and the time-dependent value of $\Omega_{m}$. Defining $\Omega_{m}(t) \equiv 8 \pi \bar{\rho}(t) / 3 M_{P}^{2} H^{2}(t)$, and using the Friedmann equation (2.8),

$$
\beta=-\frac{1+\Omega_{m}^{2}}{1-\Omega_{m}^{2}}, \quad \epsilon=\frac{8}{9} \frac{\left(1+\Omega_{m}\right)^{2}}{\left(1+\Omega_{m}^{2}\right)^{2}} \Omega_{m} \delta .
$$

We stress that $\Omega_{m}$ is a time-dependent quantity-it goes to unity at high redshift $1 \ll z \ll z_{e q}$, where the evolution is Einstein-de Sitter (but in the matter dominated regime), and at present it reduces to the usual value that we denote as $\Omega_{m}^{0}=\Omega_{m}(z=0)$. We see that $\beta$ is negative, of order unity at present, and approaches minus infinity at high redshift, whereas $\epsilon$ is proportional to $\Omega_{m} \delta$ with a coefficient of order unity. Note that for large $\delta$, Eq. (3.1) reduces to the standard evolution of spherical perturbations in general relativity. However, when $\delta$ is small, the correction term in the square brackets can be noticeably different from unity.

\section{B. Linear growth}

Let us focus first on linear perturbation growth at scales $r \ll H^{-1}$. In this regime $\delta(r, t) \ll 1$, therefore one is always in the weak-brane regime, ${ }^{2} r \gg r_{*}$, and the only effect of DGP gravity is a modification of Newton's constant. Equation (3.1) reduces to

$$
\ddot{\delta}+2 H \dot{\delta}=4 \pi G \bar{\rho}\left(1+\frac{1}{3 \beta}\right) \delta .
$$

Note that the effective Newton's constant,

$$
G_{\mathrm{eff}}=G\left(1+\frac{1}{3 \beta}\right),
$$

is time dependent. Since $\beta$ is negative, as time goes on the effective gravitational constant decreases, and this extra repulsion (compared to general relativity) leads to suppressed growth. For example, if $\Omega_{m}^{0}=0.3, G_{\text {eff }} / G=0.72,0.86,0.92$ at $z=0,1,2$.

The growing-mode solution of Eq. (3.3), $D_{+}$, is shown as a function of redshift $z$ in Fig. 1. The top panel shows as dashed lines the ratio of $D_{+}$in DGP gravity to that in a dark energy (DE) scenario with the same Friedmann equation but standard gravity, for two values of the present matter density $\Omega_{m}^{0}=0.3$ (top) and $\Omega_{m}^{0}=0.2$ (bottom). Notice how the change in the effective Newton constant leads to a suppression of $D_{+}$. Incidentally, this suppression is about two times larger than for models of modified gravity (with the same expansion history) that obey the Birkhoff's law $[15,16]$. The lower panel compares the growth factor $D_{+}$to that in the standard cosmological constant scenario (with $\Omega_{m}^{0}=0.3$ and $\Omega_{\Lambda}=0.7$ ), again for $\Omega_{m}^{0}=0.3$ (top) and $\Omega_{m}^{0}=0.2$ (bottom). We see here that the change in the expansion history (from a cosmological constant to DGP) leads to an additional suppression of the growth. In the language of dark energy, this is because the nonstandard term in the Friedmann equation (2.8) can be thought of as a contribution from a dark energy component with an effective equation of state given by

$$
w_{\mathrm{eff}}=-\frac{1}{1+\Omega_{m}},
$$

therefore, for fixed $\Omega_{m}^{0}$ such a term dominates the expansion of the Universe earlier in DGP gravity than in DE models with a cosmological constant, leading to an enhanced expan-

\footnotetext{
${ }^{2}$ This is always true for top-hat perturbations, but in practice the size $r$ is related to the amplitude $\delta$ through the perturbation spectrum. However, for $1 \sigma$ fluctuations of scale $r=10-100 \mathrm{Mpc} / h$ with typical profiles given by the two-point correlation function, $r_{*}$ corresponds to $5-15 \mathrm{Mpc} / h$. Therefore perturbations accessible to large-scale structure surveys are a natural probe of DGP gravity in the weak-brane regime.
} 


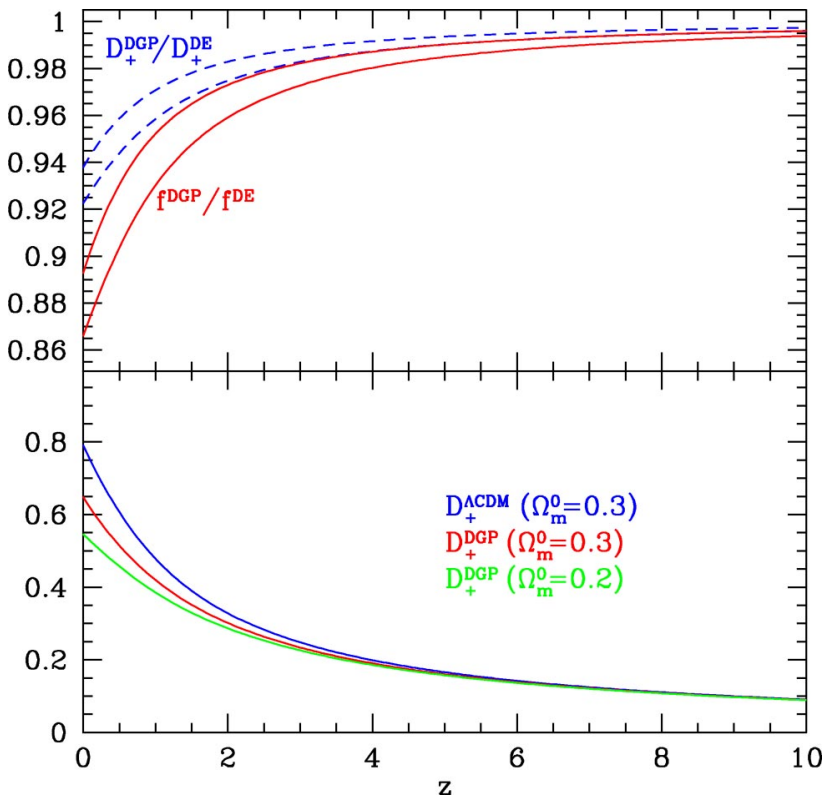

FIG. 1. The top panel shows the ratio of the growth factors $D_{+}$ (dashed lines) in DGP gravity [Eq. (3.3)] and a model of dark energy (DE) with an equation of state such that it gives rise to the same expansion history [i.e. given by Eq. (2.8), but where the force law is still given by general relativity]. The upper line corresponds to $\Omega_{m}^{0}=0.3$, the lower one to $\Omega_{m}^{0}=0.2$. The solid lines show the analogous result for velocity perturbations factors $f$. The bottom panel shows the growth factors as a function of redshift for models with different expansion histories, corresponding to (from top to bottom) $\Lambda \mathrm{CDM}\left(\Omega_{m}^{0}=0.3\right)$, and DGP gravity with $\Omega_{m}^{0}=0.3,0.2$ respectively.

sion rate $H$ and therefore an additional suppression over the one provided by the change in the force law. We will examine the observational consequences of this in Sec. IV.

The growth of velocity perturbations is also a useful observable, and it follows directly from the continuity equation in the linear approximation. It is specified by $f$ $\equiv d \ln D_{+} / d \ln a$ and it can be parametrized in terms of the time variable $\Omega_{m}$; in fact, one finds the following differential equation for $f\left(\Omega_{m}\right)$ directly from Eq. (3.3):

$$
\begin{aligned}
& -\frac{d f}{d \Omega_{m}}+\frac{1}{3 \Omega_{m}\left(1-\Omega_{m}\right)}\left[\left(2-\Omega_{m}\right) f+\left(1+\Omega_{m}\right) f^{2}\right] \\
& \quad=\frac{1}{3} \frac{\left(1+\Omega_{m}\right)\left(1+2 \Omega_{m}^{2}\right)}{\left(1-\Omega_{m}\right)\left(1+\Omega_{m}^{2}\right)}
\end{aligned}
$$

whose numerical solution follows approximately $f\left(\Omega_{m}\right)$ $\simeq \Omega_{m}^{2 / 3}$, which can be contrasted with the standard $f\left(\Omega_{m}\right)$ $\simeq \Omega_{m}^{5 / 9}$ for flat models with a cosmological constant. The top panel of Fig. 1 shows the ratio of $f$ for DGP and DE models with the same expansion history, for $\Omega_{m}^{0}=0.3,0.2$, showing that differences of at least $10 \%$ are expected, whereas comparing DGP to cosmological constant models with the same $\Omega_{m}^{0}$ larger differences are obtained, e.g. for $\Omega_{m}^{0}=0.2$, $f^{\mathrm{DGP}} / f^{\Lambda \mathrm{CDM}}=0.83$. These deviations are well within the range that can be probed with current redshift surveys.

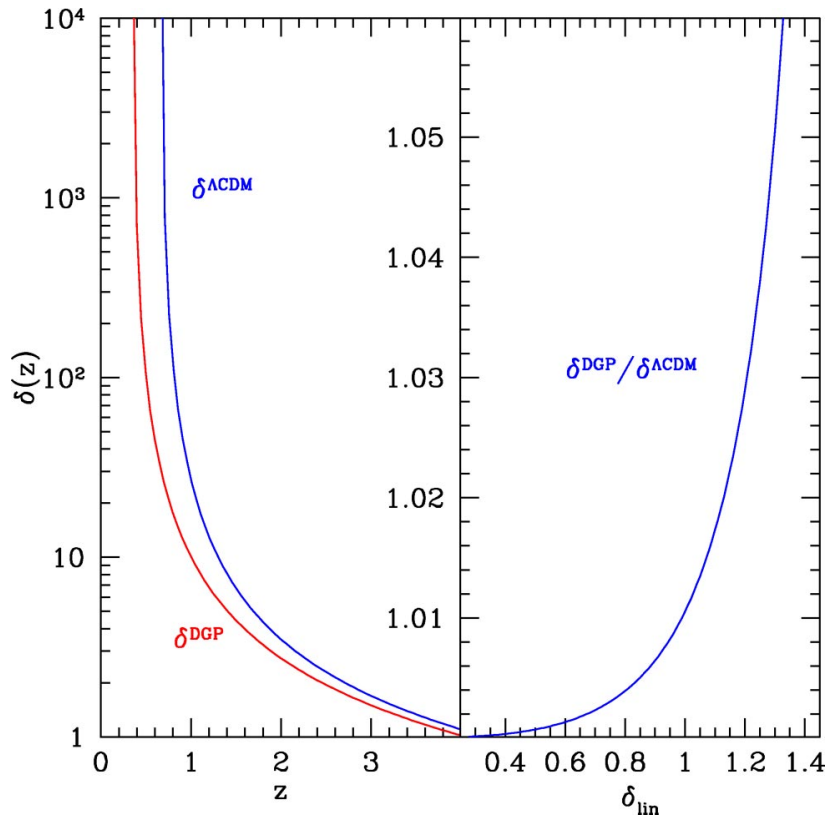

FIG. 2. Numerical solution of the spherical collapse. The left panel shows the evolution for a spherical perturbation with $\delta_{i}=3$ $\times 10^{-3}$ at $z_{i}=1000$ for $\Omega_{m}^{0}=0.3$ in DGP gravity and in $\Lambda$ CDM. The right panel shows the ratio of the solutions once they are both expressed as a function of their linear density contrasts.

\section{Nonlinear growth}

The left panel in Fig. 2 shows the full solution of Eq. (3.1) with an initial condition of $\delta_{i}=3 \times 10^{-3}$ at $z_{i}=1000$ for $\Omega_{m}^{0}=0.3$, and the corresponding solution in the cosmological constant case. Whereas such a perturbation collapses in the $\Lambda \mathrm{CDM}$ case at $z=0.66$ when its linearly extrapolated density contrast is $\delta_{c}=1.689$, for the DGP case the collapse happens much later at $z=0.35$ when its $\delta_{c}=1.656$. In terms of the linearly extrapolated density contrasts things do not look very different, in fact, when the full solutions are expressed as a function of the linearly extrapolated density contrasts, $\delta_{\operatorname{lin}}=D_{+} \delta_{i} /\left(D_{+}\right)_{i}$ they are very similar to within a few percent (right panel in Fig. 2). This implies that all the higher-order moments of the density field will be very close to that in $\Lambda \mathrm{CDM}$ models. Indeed, such moments are determined by the vertices $\nu_{n}$ defined from $\left(\nu_{1} \equiv 1\right)$

$$
\delta\left(\delta_{\text {lin }}\right)=\sum_{n=1}^{\infty} \frac{\nu_{n}}{n !} \delta_{\text {lin }}^{n},
$$

e.g., the skewness is $S_{3}=3 \nu_{2}$ [32], up to smoothing corrections that depend on the transformation from Lagrangian to Eulerian space. However since $\nu_{n}=d^{n} \delta / d \delta_{\operatorname{lin}}^{n}$ at $\delta_{\text {lin }}=0$, the $\nu_{n}$ 's in DGP gravity will all be very similar to those in $\Lambda \mathrm{CDM}$ (we have checked this explicitly for $S_{3}$, obtaining less than $1 \%$ change). This can be useful in the sense that it allows the use of the nonlinear growth to constrain the bias between galaxies and dark matter in the same way as it is done in standard case, thus inferring the linear growth factor from the normalization of the power spectrum in the linear regime. Although the result in the right panel in Fig. 2 may 
seem a coincidence at first sight, Eq. (3.1) says that the nontrivial correction from DGP gravity in square brackets is maximum when $\delta=0$ (which gives the renormalization of Newton's constant). As $\delta$ increases the correction disappears (since DGP becomes Einstein at high densities), so most of the difference between the two evolutions happens in the linear regime, which is encoded in the linear growth factor.

\section{Late-time ISW effect}

We now consider the late-time integrated Sachs-Wolfe (ISW) effect on the cosmic microwave background (CMB) for perturbations with scale $r \ll H^{-1}$. For this purpose, we need to identify the gravitational potentials for linear overdensities as perturbations around a homogeneous cosmological background with the line element

$$
\begin{aligned}
d s^{2}= & {[1+2 \Phi(\tau, \lambda)] d \tau^{2} } \\
& -\bar{a}^{2}(\tau)[1+2 \Psi(\tau, \lambda)]\left[d \lambda^{2}+\lambda^{2} d \Omega\right] .
\end{aligned}
$$

Here $\Phi(\tau, \lambda)$ and $\Psi(t, \lambda)$ are the relevant gravitational potentials and $\lambda$ is a comoving radial coordinate. In effect we want to determine $\Phi$ and $\Psi$ given $n$ and $a$. Unlike the case of Einstein's gravity, $\Phi \neq-\Psi$, due to additional contribution of the first term in Eq. (2.4). One may perform a coordinate transformation to determine that relationship. We find that, assigning $r=\bar{a}(\tau) \lambda$, and

$$
\begin{aligned}
& \Phi=n-\left.n\right|_{\text {background }} \\
& \Psi=-\int \frac{d r}{r}\left[a(\tau, r)-\left.a(\tau, r)\right|_{\text {background }}\right],
\end{aligned}
$$

keeping only the important terms when $r H \ll 1$. But since we are concerned with linear density perturbations, we find from Eqs. (2.23) and (2.24) that the quantity of interest for the ISW effect is the time derivative of

$$
\nabla^{2}(\Phi-\Psi)=\frac{8 \pi}{M_{P}^{2}} \bar{a}^{2} \rho \delta
$$

where $\nabla$ is the gradient in comoving spatial coordinates. This result is identical to the four-dimensional Einstein result, the contributions from the brane effects exactly cancelling. This result is not entirely surprising since the effect of the brane is the introduction of a gravitational scalar that couples to the trace of the energy-momentum tensor. However, the ISW effect has to do with the evolution of photons through a gravitational field (in the weak field limit), and photons will not couple to the gravitational scalar (its trace vanishes). Thus, the late-time ISW effect for DGP gravity will be identical to that of a dark energy cosmology that mimics the DGP cosmic expansion history, Eq. (2.8), at least at scales small compared to the horizon. Our approximation does not allow us to address the ISW effect at the largest scales (relevant for the CMB at low multipoles), but it is applicable to the cross correlation of the CMB with galaxy surveys. At larger scales, one expects to encounter difficulties associated with leakage of gravity off the brane (for order-unity redshifts) and other bulk effects $[30,29]$ that we were successfully able to ignore at subhorizon scales.

Discussion of photon geodesics naturally leads one to ask how lensing may be altered due to DGP contributions. For weak lensing by large-scale structure, one is in the weak field limit and therefore Eq. (3.11) applies; that is, the weak lensing pattern is identical to that for Einstein gravity, apart from the difference in expansion histories and change in the force law. In other words, reconstruction of the dark matter distribution in DGP from weak lensing only requires changing the growth rate and the geometrical distances. ${ }^{3}$

\section{E. Beyond isolated spherical perturbations}

Since we have derived the growth of spherical isolated perturbations, it is fair to ask how well do we expect our results to hold in the realistic case of a superposition of perturbations of arbitrary shape. In the linear regime, one expects to recover the same result as here, since the linearized equations obey the superposition principle and one may construct arbitrary perturbations from a linear superposition of isolated spherical perturbations. In the linear regime, DGP gravity reduces to a Brans-Dicke theory with a slowly timedependent Newton's constant, Eq. (3.4). The Newtonian potential is then just a solution to Poisson's equation for a given matter distribution source. It would be interesting, however, to corroborate this prescription with a fully consistent linear solution for an arbitrary perturbation spectrum along the lines of those presented in Ref. [30], restricted to scales smaller than the Hubble radius.

For the nonlinear growth, the situation is more complicated since the relation between the gravitational potential and the density fluctuation $\delta$ is nonlinear and the principle of superposition no longer holds. Here, however, one should keep in mind that these nonlinearities only develop late in the evolution after the universe starts to accelerate, thus the corrections to superposition have a small time to act. It is however difficult to say something more quantitative at this point.

\section{OBSERVATIONAL CONSEQUENCES}

What are the implications of these results for testing DGP gravity using large-scale structure? A clear signature of DGP gravity is the suppressed (compared to $\Lambda \mathrm{CDM}$ ) growth of perturbations in the linear regime due to the different expansion history and the addition of a repulsive contribution to the force law. However, in order to predict the present normalization of the power spectrum at large scales, we need to know the normalization of the power spectrum at early times

\footnotetext{
${ }^{3}$ It is a nontrivial result that light deflection by a compact spherical source is identical to that in four-dimensional Einstein gravity [even with potentials Eqs. (2.16)-(2.19) substantially differing from those of Einstein gravity] through the nonlinear transition between the Einstein phase and the weak-brane phase. As such, there remains the possibility that for aspherical lenses that this surprising null result does not persist through that transition and that DGP may manifest itself through some anomalous lensing feature.
} 


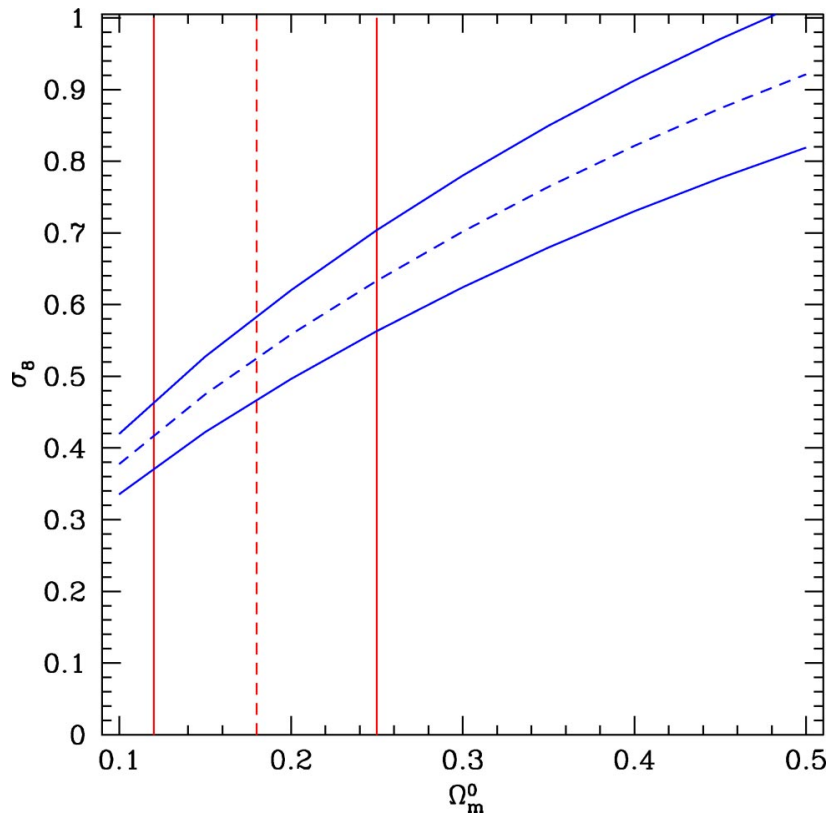

FIG. 3. The linear power spectrum normalization, $\sigma_{8}$, for DGP gravity as a function of $\Omega_{m}^{0}$. The vertical lines denote the best fit value and $68 \%$ confidence level error bars from fitting to type-IA supernovae data from [21], $\Omega_{m}^{0}=0.18_{-0.06}^{+0.07}$. The other lines correspond to $\sigma_{8}$ as a function of $\Omega_{m}^{0}$ obtained by evolving the primordial spectrum as determined by WMAP by the DGP growth factor. See text for details.

from the CMB. A fit of the pre-Wilkinson Microwave Anisotropy Probe (WMAP) CMB data was performed in Ref. [21] using the angular diameter distance for DGP gravity, finding a best fit (flat) model with $\Omega_{m}^{0} \simeq 0.3$, with a very similar CMB power spectrum to the standard cosmological constant model (with $\Omega_{m}^{0} \simeq 0.3$ and $\Omega_{\Lambda}^{0}=0.7$ ) and other parameters kept fixed at the same value. Here we use this fact, plus the normalization obtained from the best-fit cosmological constant power-law model from WMAP [33] which has basically the same (relevant for large-scale structure) parameters as in Ref. [21], except for the normalization of the primordial fluctuations which has increased compared to preWMAP data (see e.g. Fig. 11 in Ref. [34]). The normalization for the cosmological constant scale-invariant model corresponds to present rms fluctuations in spheres of $8 \mathrm{Mpc} / h$, $\sigma_{8}=0.9 \pm 0.1$ (see Table 2 in Ref. [33]). We assume a flat model, since it was shown in Ref. [21] to be consistent as well with the DGP angular diameter distance. We ignore the fact that the ISW effect at low multipoles (where the CMB power spectrum has large error bars) can be different in DGP gravity, this has a small effect on the overall normalization of the primordial fluctuations that are determined by the overall power spectrum.

Figure 3 shows the present value of $\sigma_{8}$ as a function of $\Omega_{m}^{0}$ for DGP gravity, where we assume that the best-fit normalization of the primordial fluctuations stays constant as we change $\Omega_{m}^{0}$, and recompute the transfer function and growth factor as we move away from $\Omega_{m}^{0}=0.3$. Since most of the contribution to $\sigma_{8}$ comes from scales $r<100 \mathrm{~h} / \mathrm{Mpc}$, we can calculate the transfer function using Einstein gravity, since these modes entered the Hubble radius at redshifts high enough that they evolve in the standard fashion. The value of $\sigma_{8}$ at $\Omega_{m}^{0}=0.3$ is then given by 0.9 times the ratio of the DGP to $\Lambda$ CDM growth factors shown in the bottom panel of Fig. 1. The error bars in $\sigma_{8}$ reflect the uncertainty in the normalization of primordial fluctuations, and we keep them a constant fraction as we vary $\Omega_{m}^{0}$ away from 0.3 . We see in Fig. 3 that for the lower values of $\Omega_{m}^{0}$ preferred by fitting the acceleration of the Universe, the additional suppression of growth plus the change in the shape of the density power spectrum drive $\sigma_{8}$ to a rather small value. This could in part be ameliorated by increasing the Hubble constant, but not to the extent needed to keep $\sigma_{8}$ at reasonable values. The vertical lines show the best-fit and $1 \sigma$ error bars from fitting DGP gravity to the supernova data from Ref. [1] in Ref. [21]. This shows that fitting the acceleration of the universe requires approximately $\sigma_{8} \leqslant 0.7$ to $1 \sigma$ and $\sigma_{8} \leqslant 0.8$ to $2 \sigma$.

In order to compare this prediction of $\sigma_{8}$ to observations one must be careful since most determinations of $\sigma_{8}$ have built in the assumption of Einstein gravity or $\Lambda$ CDM models. We use galaxy clustering, which in view of the results in Sec. III C for higher-order moments, should provide a test of galaxy biasing independent of gravity being DGP or Einstein. Recent determinations of $\sigma_{8}$ from galaxy clustering in the SDSS survey [35] give $\sigma_{8}^{*}=0.89 \pm 0.02$ for $L^{*}$ galaxies at an effective redshift of the survey $z_{s}=0.1$. We can convert this value to $\sigma_{8}$ for dark matter at $z=0$ as follows. We evolve to $z=0$ using a conservative growth factor, that of DGP for $\Omega_{m}^{0}=0.2$. In order to convert from $L^{*}$ galaxies to dark matter, we use the results of the bispectrum analysis of the $2 \mathrm{dF}$ survey [36] where $b=1.04 \pm 0.11$ for luminosity $L$ $\simeq 1.9 L^{*}$. We then scale to $L^{*}$ galaxies using the empirical relative bias relation obtained in Ref. [37] that $b / b^{*}=0.85$ $+0.15\left(L / L^{*}\right)$, which is in very good agreement with SDSS (see Fig. 30 in Ref. [35]). This implies $\sigma_{8}=1.00 \pm 0.11$. Even if we allow for another $10 \%$ systematic uncertainty in this procedure, the preferred value of $\Omega_{m}^{0}$ in DGP gravity that fits the supernovae data is about $2 \sigma$ away from that required by the growth of structure at $z=0$.

An independent way of testing DGP gravity with largescale structure is to constrain the growth of velocity fluctuations through $f$. This affects the redshift distortions of the power spectrum and can be extracted from measurements, though at present the errors are somewhat large (see, e.g., Ref. [35]) for an accurate test, but this should improve soon. The interesting feature of this test is that it is independent of the normalization of the primordial fluctuations, unlike the $\sigma_{8}$ normalization discussed above; thus it will be important to check that the same conclusions follow in this case.

\section{CONCLUDING REMARKS}

In this paper we identified how one may test the modifications of the gravitational force law expected in DvaliGabadadze-Porrati (DGP) gravity at scales of cosmological interest. While cosmology is altered when the Hubble scale becomes comparable to today's Hubble scale, $H_{0}^{-1}$, the force law is correspondingly altered at much shorter scales and affects, for example, the growth of density perturbations at redshifts of order unity. 
Although the results obtained in this paper are qualitatively comparable to those found in Ref. [15], they differ in key ways since DGP gravity does not obey Birkhoff's law. The form of the gravitational force law between localized mass sources is sensitive to the background cosmological expansion. So, while the results show deviations from Newtonian gravity of order unity at distance scales greater than the scale $r_{*} \sim\left(r_{g} / H_{0}^{2}\right)^{1 / 3}$, where $r_{g}$ is the Schwarzschild radius of the mass source, the quantitative details differ. In particular, the suppression of the growth of structure is a factor about 2 larger than in Birkhoff-law models with the same expansion history. Moreover, DGP gravity deviates significantly from four-dimensional Einstein gravity through the emergence of an ultralight graviscalar mode. Because such a mode does not couple to photons, the effects do not manifest themselves in the late-time integrated Sachs-Wolfe (ISW) effect, at least at the subhorizon scales we consider. While the gravitational potentials are indeed altered by order-unity factors late in the cosmic expansion history, they precisely cancel, so that the late-time ISW effect in DGP gravity is identical to that for a dark energy theory that mimics the DGP expansion history. The same situation applies to weak gravitational lensing.

We have done a first assessment of the observational viability of DGP gravity to simultaneously explain the acceleration of the Universe and the growth of structure. In order to improve the comparison against observations a number of issues remain unsolved. First, one would like to check that the linear growth factor for subhorizon scales derived under the spherical approximation holds for more general perturbations, as expected by the superposition principle in the linear regime. A more nontrivial check would be to generalize this to the nonlinear case, or at least second-order in perturbation theory, to check the deviations from superposition assumed here in the nonlinear case. To do a full comparison of the CMB power spectrum against data it remains to solve for the ISW effect at scales comparable to the horizon. Our treatment found no difference from general relativity (except from the change in the expansion history), but this is only valid at subhorizon scales.

Nevertheless, the main problem for DGP gravity to simultaneously explain cosmic acceleration and the growth of structure is easy to understand: the expansion history is already significantly different from a cosmological constant, corresponding to an effective equation of state with $w_{\text {eff }}$ $=-\left(1+\Omega_{m}\right)^{-1}$. This larger value of $w$ suppresses the growth somewhat due to earlier epoch of the onset of acceleration. In addition, the repulsive contribution to the force law suppresses the growth even more, driving $\sigma_{8}$ to a rather low value, in contrast with observations. If as error bars shrink the supernovae results continue to be consistent with $w_{\text {eff }}=-1$, this will drive the DGP fit to a yet lower value of $\Omega_{m}^{0}$ and thus a smaller value of $\sigma_{8}$. For these reasons we expect the tension between explaining acceleration and the growth of structure to be robust to a more complete treatment of the comparison of DGP gravity against observations.

\section{ACKNOWLEDGMENTS}

The authors thank C. Deffayet, G. Dvali, G. Gabadadze, A. Gruzinov, M. Takada, and M. Zaldarriaga for helpful communications and insights. A.L. and G.S. wish to thank the CERN Theory Division and the Center for Cosmology and Particle Physics for their hospitality. This work was sponsored by DOE Grant DEFG0295ER40898, the CWRU Office of the Provost, NASA grant NAG5-12100, NSF grant PHY-0101738, and CERN. G.S. thanks Maplesoft for the use of Maple V.
[1] Supernova Cosmology Project Collaboration, S. Perlmutter et al., Astrophys. J. 517, 565 (1999).

[2] Supernova Search Team Collaboration, A.G. Riess et al., Astron. J. 116, 1009 (1998).

[3] C. Deffayet, Phys. Lett. B 502, 199 (2001).

[4] C. Deffayet, G.R. Dvali, and G. Gabadadze, Phys. Rev. D 65, 044023 (2002).

[5] T. Damour, I.I. Kogan, and A. Papazoglou, Phys. Rev. D 66, 104025 (2002).

[6] K. Freese and M. Lewis, Phys. Lett. B 540, 1 (2002).

[7] K. Freese, Nucl. Phys. B (Proc. Suppl.) 124, 50 (2003).

[8] G. Dvali and M.S. Turner, astro-ph/0301510.

[9] S. Capozziello, S. Carloni, and A. Troisi, astro-ph/0303041.

[10] S.M. Carroll, V. Duvvuri, M. Trodden, and M.S. Turner, astro-ph/0306438.

[11] S. Capozziello, V.F. Cardone, S. Carloni, and A. Troisi, Int. J. Mod. Phys. D 12, 1969 (2003).

[12] S. Nojiri and S.D. Odintsov, Phys. Rev. D 68, 123512 (2003).

[13] J. Khoury and A. Weltman, astro-ph/0309300.

[14] J. Khoury and A. Weltman, Phys. Rev. D 69, 044026 (2004).

[15] A. Lue, R. Scoccimarro, and G. Starkman, Phys. Rev. D 69, 044005 (2004).
[16] T. Multamaki, E. Gaztanaga, and M. Manera, Mon. Not. R. Astron. Soc. 344, 761 (2003).

[17] G. Dvali, G. Gabadadze, and M. Porrati, Phys. Lett. B 485, 208 (2000).

[18] G.R. Dvali, G. Gabadadze, M. Kolanovic, and F. Nitti, Phys. Rev. D 64, 084004 (2001).

[19] G.R. Dvali, G. Gabadadze, M. Kolanovic, and F. Nitti, Phys. Rev. D 65, 024031 (2002).

[20] P.P. Avelino and C.J.A. Martins, Astrophys. J. 565, 661 (2002); C. Deffayet, G.R. Dvali, and G. Gabadadze, astro-ph/0106449.

[21] C. Deffayet, S.J. Landau, J. Raux, M. Zaldarriaga, and P. Astier, Phys. Rev. D 66, 024019 (2002).

[22] J.S. Alcaniz, Phys. Rev. D 65, 123514 (2002); D. Jain, A. Dev, and J.S. Alcaniz, ibid. 66, 083511 (2002); J.S. Alcaniz, D. Jain, and A. Dev, ibid. 66, 067301 (2002).

[23] C. Deffayet, G.R. Dvali, G. Gabadadze, and A.I. Vainshtein, Phys. Rev. D 65, 044026 (2002).

[24] A. Lue, Phys. Rev. D 66, 043509 (2002).

[25] A. Gruzinov, astro-ph/0112246.

[26] M. Porrati, Phys. Lett. B 534, 209 (2002).

[27] A. Lue and G. Starkman, Phys. Rev. D 67, 064002 (2003). 
[28] G. Dvali, A. Gruzinov, and M. Zaldarriaga, Phys. Rev. D 68, 024012 (2003).

[29] A. Lue, Phys. Rev. D 67, 064004 (2003).

[30] C. Deffayet, Phys. Rev. D 66, 103504 (2002).

[31] M.A. Luty, M. Porrati, and R. Rattazzi, J. High Energy Phys. 09, 029 (2003).
[32] F. Bernardeau, Astrophys. J. 392, 1 (1992).

[33] D.N. Spergel et al., Astrophys. J., Suppl. Ser. 148, 175 (2003).

[34] G. Hinshaw et al., Astrophys. J., Suppl. Ser. 148, 135 (2003).

[35] M. Tegmark et al., astro-ph/0310725.

[36] L. Verde et al., Mon. Not. R. Astron. Soc. 335, 432 (2002).

[37] P. Norberg, Mon. Not. R. Astron. Soc. 328, 64 (2001). 